\title{
TRICHILEMMAL CYSTIS IN METASTATIC MELANOMA: A CASE REPORT
}

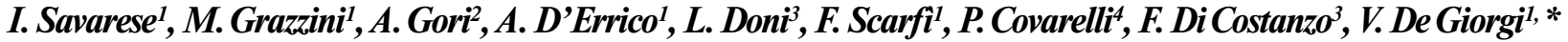 \\ ${ }^{1}$ Department of Dermatology, Department of Critical Care Medicine and Surgery, University of Florence, \\ Firenze 50121, 50132, Italy \\ ${ }^{2}$ Cancer Research "Attilia Pofferi” Foundation, Pistoia 51100, Italy \\ ${ }^{3}$ Oncology Unit, Department of Critical Care Medicine and Surgery, University of Florence, \\ Firenze 50121, 50132, Italy \\ ${ }^{4}$ Department of Surgery, University of Perugia, Perugia 06121, Italy
}

The malignant melanoma is a neoplasm associated with a wide variety of cutaneous paraneoplastic syndromes, as dermatomyositis, systemic sclerosis, paraneoplastic pemphigus. We describe a case of four multiple trichilemmal cystis arising on frontal region in the same patient with brain metastasis and unknown primary melanoma and discuss their relationship.

Key Words: paraneoplastic syndromes, melanoma, trichilemmal cystis, metastasis.

Skin can be a clue of systemic diseases like malignancy. In paraneoplastic disorders, cutaneous manifestations generally take place at a distance from the primary tumor site. The malignant melanoma is a neoplasm associated with a wide variety of cutaneous paraneoplastic syndromes, as dermatomyositis [1], systemic sclerosis [2], paraneoplastic pemphigus [3].

We describe a case of four multiple trichilemmal cystis (TCs) arising on frontal region in the same patient with brain metastasis and unknown primary melanoma and discuss their relationship.

In September 2011, a 57-year-old man was referred to our Department of Dermatology, University of Florence. The previous months, the patient had a history of neurologic signs (cephalea and left foot paralysis) evaluated further with instrumental diagnostic. Brain CT scan showed an expansive lesion, $43 \times 18 \mathrm{~mm}$ of size, in parafalcal frontal region of right cerebral hemisphere, leading to a complete excision and confirmation of further malignant melanoma cerebral metastasis. Subsequently patient underwent instrumental investigation as digestive endoscopy, brain magnetic resonance imaging and total body computed tomography scan for searching the primary melanoma and/or lymphonodal or visceral metastasis. Neither primary nor secondary tumor was detected. On clinical examination, in search of primary melanoma or any cutaneous melanoma metastasis, we found 4 pigmented lesions on the frontal region. The patient has noticed a recent appearance of these lesions 3 months before (almost simultaneously with neurological disorders), but disregarded them. Clinically lesions seemed welldemarcated, partially indurated, blue nodules, with a diameter of $2 \times 3 \mathrm{~mm}$ each one. On dermoscopical examination we observed diffuse homogeneous bluegrey pigmentation (Figure).

Submitted: October 04, 2016.

*Correspondence: E-mail: vincenzo.degiorgi@unifi.it

Tel.: 0039-055-6939632;

fax: 0039-055-6939632

Abbreviation used: TCs - trichilemmal cystis.
Although homogeneous blue pattern on dermoscopy is a feature of blue nevus, in this case regarding anamnesis and sudden eruption, we advanced diagnosis of cutaneous melanoma metastasis. All lesions were excised and histopathologically a final diagnosis of TCs was done. In November, the patient began head and neck radiotherapy (30 Gy). Positron emission tomography scan did not point out any primary and/ or secondary malignancy. On clinical and dermatoscopic examination, no suspicious lesions or palpable lymphadenopathy were detectable, so we proposed a follow-up every 3 months. Nine months after surgery, no metastasis were found clinically and/or in image studies.

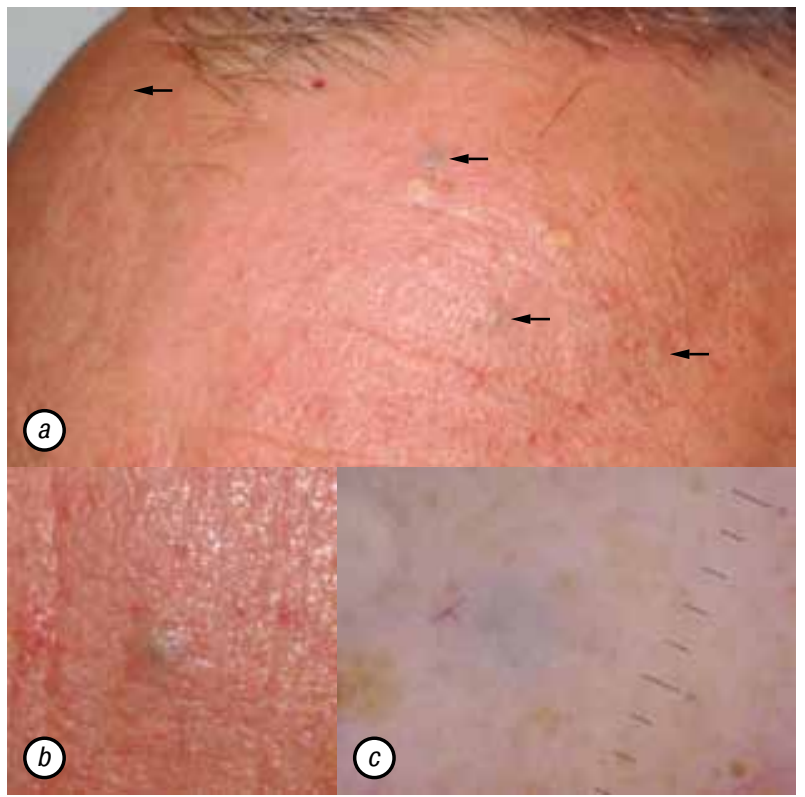

Figure. A case of TCs in metastatic melanoma: a - clinically, well-demarcated, round, partially indurated, blue color nodules, $2 \times 3 \mathrm{~mm}$ in diameter each, are demonstrated in frontal region; $b$ - one of four blue nodules is shown; $c$ - on dermoscopical examination, homogeneous blue-grey diffuse pigmentation, arborizing vessels and solar lentigines around the lesion are visible

We discuss a possible relationship between TCs and melanoma in a patient with brain metastasis and unknown primary melanoma. 
In literature many cutaneous paraneoplastic syndromes are described in association with melanoma [1-3]. In these paraneoplastic disorders, the skin condition generally is a consequence of cancer, but is not due to the local presence of cancer cells as such. According to Curth $[4,5]$, criteria that define a paraneoplastic cutaneous syndrome include: (1) development of a dermatosis only after the development of a malignant neoplasm, (2) both the dermatosis and the malignant neoplasm follow a parallel clinical course, (3) the condition is not recognized as part of a genetic syndrome, (4) a specific tumor occurs with a probable dermatosis, (5) the dermatosis is not common, and (6) a high percentage of the association is noted. A harmonized ethiology for paraneoplastic cutaneous manifestations is doubtful. It is hypothesized that mediators such as growth factors, cytokines, active hormones and other unidentified mediators by tumor or tumor-induced antigen-antibody interactions are involved in the pathogenesis of the cutaneous findings. Among cutaneous paraneoplastic manifestations, pigmentary disorders were described in literature $[6,7]$.

In our case, the fast and sudden eruption of pigmented lesions occurred during the period of first neurological signs. No association between TCs and neoplasm was described in literature. Instead, multiple trichilemmomas can be associated to neoplasm as melanoma in Cowden syndrome. Trichilemmal (or pylar) cyst, as trichilemmoma, origins from the "trichilemma" or the exposed outer root sheath. Clinically TCs appear as asymptomatic swellings nodules, typically without visible pores. On dermoscopy, TCs can appear with homogeneous blue pattern, probably due to keratinous mass according to Gencoglan et al. [8], and simulate cutaneous melanoma metastasis.

The temporal relationship of the cutaneous eruption of TCs with advanced stage of melanoma (TxNOM1c) in our patient strongly suggests a probable paraneoplastic manifestation. While in our case primary melanoma is still to be discovered, the mediators released by melanoma could be implicated in TC onset.

\section{REFERENCES}

1. Tu J, Von Nida J. Metastatic malignant melanoma and dermatomyositis: A paraneoplastic phenomenon. Australas J Dermatol 2011; 52: e7-10.

2. Thoelke A, Schmid HP, Figl R, et al. Jo-1 positive paraneoplastic systemic sclerosis in a patient with metastatic melanoma. Eur J Dermatol 2006; 16: 428-30.

3. Tilakaratne W, Dissanayake M. Paraneoplastic pemphigus: a case report and review of literature. Oral Dis 2005; 11: $326-9$.

4. Curth HO. Skin lesions and internal carcinoma. In: Andrade R, Gumport SL, Popkin GL, Reed TD, eds. Cancer of the skin: biology, diagnosis, management. Philadelphia: WB Saunders, 1976: 1308-43.

5. McLean DI. Cutaneous paraneoplastic syndromes. Arch Dermatol 1986; 122: 765-7.

6. Brenner S, Tamir E, Maharshak N, Shapira J. Cutaneous manifestations of internal malignancies. Clin Dermatol 2001; 19: 290-7.

7. Gass JD, Glatzer RJ. Acquired pigmentation simulating Peutz - Jeghers syndrome: initial manifestation of diffuse uveal melanocytic proliferation. Br J Ophthalmol 1991; 75: 693-5.

8. Gencoglan G, Karaarslan IK, Akalin T, Ozdemir F. Trichilemmal cyst with homogeneous blue pigmentation on dermoscopy. Australas J Dermatol 2009; 50: 301-2. 